NBER WORKING PAPER SERIES

\title{
DIFFERENTIATION STRATEGY AND MARKET DEREGULATION: \\ LOCAL TELECOMMUNICATION ENTRY IN THE LATE 1990s
}

\author{
Shane Greenstein \\ Michael Mazzeo \\ Working Paper 9761 \\ http://www.nber.org/papers/w9761 \\ NATIONAL BUREAU OF ECONOMIC RESEARCH \\ 1050 Massachusetts Avenue \\ Cambridge, MA 02138 \\ June 2003
}

We thank the Searle Foundation and Kellogg School of Management for funding. We thank Greg Rosston, Bill Rogerson, Glenn Woroch and Dennis Yao for many useful remarks, as well as seminar participants at Carnegie-Mellon, Cornell, Syracuse and Washington Universities, the NBER IO Winter 2003 meetings and the Conference on Management Strategy and the Business Environment at the Harvard Business School. Avi Goldfarb provided excellent research assistance. We are grateful to Greg Rosston and Brad Wimmer for providing us data on ILECs. All errors are our responsibility. The views expressed herein are those of the authors and not necessarily those of the National Bureau of Economic Research.

(C)2003 by Shane Greenstein and Michael Mazzeo. All rights reserved. Short sections of text not to exceed two paragraphs, may be quoted without explicit permission provided that full credit including (C) notice, is given to the source. 
Differentiation Strategy and Market Deregulation:

Local Telecommunication Entry in the Late 1990s

Shane Greenstein and Michael Mazzeo

NBER Working Paper No. 9761

June 2003

JEL No. L5, L9

\begin{abstract}
The authors examine the role of differentiation strategies for entry behavior in markets for local telecommunication services in the late 1990s. Whereas the prior literature has used models of interaction among homogenous firms, this research is motivated by the claim of entrants that they differ substantially in their product offerings and business strategies. Exploiting a new, detailed data set of Competitive Local Exchange Carriers (CLECs) entry into over 700 U.S. cities, the authors take advantage of recent developments in the analysis of entry and competition among differentiated firms. They test and reject the null hypothesis of homogeneous competitors. They also find strong evidence that CLECs account for both potential market demand and the business strategies of competitors when making their entry decisions. This suggests that firms' incentives to differentiate their services should shape the policy debate for competitive local telecommunications.
\end{abstract}

Shane Greenstein

J.L. Kellogg School of Management Northwestern University

2001 Sheridan Road

Evanston, IL 60208-2013

and NBER

greenstein@kellogg.northwestern.edu
Michael Mazzeo

J.L. Kellogg School of Management Northwestern University

2001 Sheridan Road

Evanston, IL 60208-2013

mazzeo@kellogg.northwestern.edu 


\section{Introduction}

By the end of 1990s many cities in the United States had experience with competitive local telephony. In many locales Competitive Local Exchange Carriers (CLECs) entered into competition with each other and with the Incumbent Local Exchange Carrier (ILEC). In 1999, CLECs accounted for over \$20 billion in annual revenue (New Paradigm Resources Group 2000). Despite their considerable size and their comparative novelty, only a few economics studies have analyzed the competitive behavior of these firms.

In this paper we consider the extent to which incentives to offer differentiated services affected the entry strategies of CLECs. We reject the null hypothesis that entry behavior is unrelated to the types of services offered by firms (i.e., we reject homogeneous competitors). Although regulators and market participants suspected that opportunities for product differentiation could arise, previous analyses have not measured differentiation explicitly nor considered its importance in market development. We argue, however, that it is central for understanding the economic and regulatory factors shaping competitive local telephony in this time period. Our analysis also sheds light on the umbrella national policy for competitive telephony, as embodied in Federal Communications Commission (FCC) rule making for the Telecommunications Act of 1996.

\section{I.i Motivation}

We consider two alternative motivations for introducing competition into industries such as local telephony. We label the first motivation scale-exceeding efficiencies. It is the predominant framework used by standard analysis (Woroch 2001) and, as far as we know, motivates all empirical models of CLEC entry to date. ${ }^{1}$ It frames

\footnotetext{
${ }^{1}$ This literature preceded the 1996 Act. Woroch (2001) provides a comprehensive review of the literature with strong emphasis on scale-exceeding efficiencies. Another variant looks at price behavior, where these prices may (or may not) reflect the ability of new entrants to price underneath regulated (i.e., artificially) high prices to business customers (e.g., Rosston and Wimmer 2001). Another variant estimates a model of market share and assumes the ILEC is first mover in a Stackleberg game with CLECs (Abel 2002). Crandall's (2001) or Crandall and Sidek's (2002)analysis is more explicit about the role of differentiation. Yet, their models of empirical revenue only partly employ insights about differentiation.
} 
analysis in terms of the costs of providing similar services in either one or more organizations. The CLEC's prospects depend on its ability to lower costs - lower than an incumbent monopolist currently achieves - with its advantages of scale and/or scope economies. As in any standard model of price competition between otherwise homogeneous services, prices fall when entrants can achieve a level of efficiency exceeding that of the incumbent.

An alternative motivation for competition is something we label customertargeted differentiation. This alternative is not reflected in existing empirical or most theoretical models. According to industry analysts and participants, CLECs have the entrepreneurial ability to identify market opportunities otherwise unmet by the ILEC. These initiatives may reflect different visions about customer needs for service or the ability of entrants to offer innovative services without the obligations (e.g., universal service and reliability) imposed on ILECs by local regulators. It may also reflect the ability of a CLEC to offer a national product on a uniform basis, something expressly impossible for many ILECs to do at this time (Shiman and Rosenworcel 2002). Price competition arises in the context of the willingness of customers to pay for the heterogeneous services. Competitive entry increases the variety available: CLECs do not pursue the same strategies, and their services are often not the same as those offered by the ILEC.

Pointed disagreements between these alternative views account for sharp differences in policy recommendations and assessment. The scale-exceeding efficiencies view would emphasize only the number of firms that have entered, that is, policy is based on simple counts of competitors. The customer-targeted differentiation view argues that models of homogenous consumers and mere counts of firms provide misleading implications about how entrants can differ and how and where consumers would value variety. Therefore, a more appropriate checklist for measuring local competition would also incorporate the types of services offered by each of the CLECs. While both views favor interconnection policies that give entrants a market test, the latter view also encourages CLEC strategies that differ from each other and those of the ILEC. 


\section{I.ii Synopsis of Model, Data, and Results}

We build on the empirical literature that analyzes entry in concentrated markets and competition among differentiated firms. As did Zolnierek, Eisner, and Burton (2001), we examine cross-sectional differences associated with the number of CLECs operating in an area. As in that study, we evaluate the impact of economic factors, such as demand and cost differences across markets, economies of geographic scope, and regulatory stringency. Unlike previous research, we tailor our estimation to small and medium-sized cities. We do this for three reasons. First, many large cities had already experienced competitive entry by the early 1990s, but it was new to medium- sized cities after the passage of the 1996 Telecommunications Act. Second, prior studies that used broader geographic market definitions provide potentially misleading insight about the extent of competition. ${ }^{2}$ Third, this focus allows us to isolate the role of product differentiation, which is difficult to measure unambiguously in large cities.

To focus the analysis on differentiated entrants, we employ an empirical framework found in Mazzeo's (2002) study that evaluates market structure in terms of both the number of firms and the types of (potentially differentiated) products that firms offer. Parameters in the model measure whether entrants behave as if competition from a similar type of firm is expected to hurt profits more than competition from one that is different. These parameters statistically test a null hypothesis of homogeneous competitors against an alternative that incorporates the differential impact of heterogeneous competitors. Applying this statistical test to the market for CLECs is the central contribution of our study.

The econometric model requires finely grained data. We employ a data set derived from the New Paradigm Resources Group (NPRG), which publishes an annual census on CLEC activity. The NPRG (2000) report tracks CLEC entry at the city level, which is the appropriate market definition, since CLECs often compete by block to-block in geographically focused areas. The NPRG report also documents differences between

\footnotetext{
${ }^{2}$ Zolneirek, Eisner, and Burton (2001) count the number of firms in each local area transport area (LATA), a wide geographic area that typically contains several cities large enough to support entry of individual CLECs. Two CLECs operating in different cities within the same LATA would not be competitors in our data. Recent FCC reports count entrants at the zip code, but they do not differentiate between type of entrant (Federal Communications Commission, 2000, 2001).
} 
the strategies of the various CLECs. This provides the data for studying the differentiation and competition hypothesis described above. The differences used in our study are simple to measure and use in policy evaluation, so we will place emphasis on demonstrating their feasibility and meaningfulness. This demonstration is another contribution of our analysis.

We use data from 1999, the last year of unambiguously optimistic growth prospects for CLECs. By 1999 there was no question that most major cities could support some CLECs in addition to the ILEC. Yet, even by the end of the 1990s competition was not widespread in many small and medium-sized cities. Because competition arose in some of these cities and not in others, data from this year expose the factors that facilitate the advance of competition in local telephony.

Our results are stark. We reject the null hypothesis of homogenous product competition. We also find that the motivation to provide heterogeneous services is comparable to other demographic and regulatory factors in terms of its contribution to observed market structure. We argue that these results support a change in the analysis and evaluation of competitive behavior and interconnection in local telephony, which elevates differentiation to a level of much greater importance. This framing also provides a number of new insights about the local demographic and regulatory factors making entry more or less attractive, details that we explore below.

\section{The Economics of CLEC Market Structure.}

An empirical analysis of CLEC entry that is motivated by scale-exceeding efficiencies has three basic elements: (1) the size of local market demand, (2) the costs of entering and operating, including potential economies of scope across markets, and (3) how demand and costs (elements 1 and 2) interact with the regulatory setting. A model of customer-targeted differentiation has those same elements, plus more. In this section, we describe the implications of each approach. Because differentiation has not been addressed explicitly in prior empirical CLEC studies, we explain why it is an important consideration, while drawing connections with previous work (e.g., Crandall 2001, Crandall and Sidek 2001, Zolnierek, Eisner and Burton 2001, Abel 2002). 


\section{II.i. Local Market Demand for Vertically Disintegrated Firms}

In traditional analyses, CLEC entry behavior is shaped by the presence of fixed costs in the provision of local telephone service. Whether they build their own facilities or lease part of their network from the ILEC, CLECs incur costs to set up and maintain the infrastructure needed to offer services. These fixed costs range from engineering costs to marketing expenses to costs associated with negotiating interconnection agreements. While some of these expenses may vary with the long-term size of the revenue stream, every CLEC incurs substantial fixed costs associated with initiating a previously unknown firm and maintaining operations of an ongoing business.

Zolnierek, Eisner and Burton (2001), following the spirit of the New Empirical Industrial Organization (NEIO) on entry (Bresnahan and Reiss (1991), Berry (1992)), hypothesize that the need to cover these fixed costs limits the number of entrants in individual markets. That is, because of the presence of fixed costs in each location where they provide services, CLECs require a sufficient level of variable profits - quantity of customers times operating margins - to cover their fixed expenses. Cities vary most notably in their size, which affects the demand for CLEC services. Operating margins may also be different across markets, particularly in cases where variable cost rates are established by local regulators. The level of competition within markets may affect operating margins as well. As a result of all of these differences, we expect the number of CLECs operating to vary across cities.

Using our data set of CLECs in Table 1, we illustrate a key part of this logic.. Among the 718 cities where CLECs have entered or made plans to enter, ${ }^{3}$ over 400 have just one CLEC and over 600 have fewer than five; on the other hand only 55 cities have ten or more CLECs and only 18 have twenty or more. As expected, cities with larger populations have the largest number of potential entrants. whereas smaller cities have the fewest. ${ }^{4}$ We will augment our market size measures beyond residential population in the empirical analysis.

\footnotetext{
${ }^{3}$ Including CLECs that are planning service in particular cities constitutes the most optimistic assessment of CLEC entry possible (we precisely define "planned" below).

${ }^{4}$ In Table 1 average population size is calculated over all the cities within a category,that is, it includes all
} 
Table 1: Sample of Cities with Specific Number of CLECs Operating or Planned

\begin{tabular}{|c|c|c|c|}
\hline $\begin{array}{c}\text { Number } \\
\text { of } \\
\text { CLECs }\end{array}$ & $\begin{array}{c}\text { Number } \\
\text { of } \\
\text { Cities }\end{array}$ & $\begin{array}{c}\text { Representative } \\
\text { Cities }\end{array}$ & $\begin{array}{c}\text { Average Population } \\
\text { Size }\end{array}$ \\
\hline $20+$ & 18 & New York, Dallas, Chicago, Atlanta & $1,438,877$ \\
\hline $10-19$ & 38 & Cleveland, St. Louis, Tucson, Norfolk & 573,492 \\
\hline $5-10$ & 54 & Little Rock, Fresno, Madison, Omaha, & 226,935 \\
\hline $3-4$ & 77 & Bakersfield, Reno, Gainesville, Waco & 158,211 \\
\hline 2 & 91 & Biloxi, Fargo, Kalamazoo, Naperville & 84,260 \\
\hline 1 & 441 & Bangor, Bismark, Champaign, Yonkers & 43,640 \\
\hline Total & 718 & & 137,143 \\
\hline
\end{tabular}

We also investigate differences across cities in the costs of providing CLEC services, including both fixed and operating costs. One source of such differences may relate to economies of scope. If two neighboring cities share economic infrastructure or have similar telecommunications demand, costs incurred by a CLEC entrant may be shared between the neighboring markets. For example, medium-sized cities near large metropolitan areas may experience more entry than do similar markets located further from a major urban hub.

More direct differences in costs may result from the regulatory environments in which CLECs enter and operate. Although the 1996 Telecommunications Act prescribed pro-competitive regulatory rules designed to foster market-oriented decision making for investment in local data and voice services, individual markets varied in how implementation of the rules affected local entrants. For example, Mini (2001) carefully documents that CLECs had distinct experiences depending on whether they were interconnecting with (1) Regional Bell Operating Companies (RBOCs), (2) GTE (renamed Verizon after its merger), or (3) another independent telecommunications firm. ${ }^{5}$ To the extent that such differences create different entry costs for CLECs, a location may be more or less attractive to CLECs.

18 cities with twenty or more entrants, all 38 with between ten and nineteen, and so on.

${ }^{5}$ RBOCs developed interconnection with entrants as part of a quid-pro-quo with the FCC, which sought to disallow entry into the long-distance market until RBOCs complied with a series of tests for opening their local markets (Shiman and Rosenwercel 2002). In contrast, the non-RBOC incumbents simply made deals under the guidance of their local state regulators. 
There is also considerable evidence of differences in the way state regulators enforced prescriptions in the 1996 Telecommunications Act, which induced differences in costs across localities. For example, policies for re-averaging prices between high business and low residential rates affected the attractiveness of providing services to business (Rosston and Wimmer 2001). Similarly, state agencies set varying wholesale prices within and across states that affected variable costs of interconnection (Gregg 2002). Other state regulatory agencies made it easy or difficult to become a seller or value-added reseller of services related to DSL. We work with the hypothesis that a state regulatory agency can make entry in particular cities more or less attractive for CLECs by affecting the fixed costs of entry as well as the variable costs of operating.

\section{II. ii Entry, Competition and Product Differentiation}

The discussion above highlights a series of market conditions that potentially influence CLEC entry, either through market size or by raising or lowering costs. If CLECs choose to enter by judging whether they expect variable profits to exceed entry costs, prices must come into play as well. Standard models predict prices will be lower in markets with more competitors - this suggests that the market size (quantity) necessary to support additional CLECs will increase as the number of operating firms increases. Bresnahan and Reiss (1991) find such a pattern between entry and market size in their study of several homogeneous service industries.

If CLECs follow a model of customer-targeted differentiation, however, the competitive effects of additional entry may be somewhat mitigated. Prices will not fall as quickly with additional entry if CLECs offer differentiated products and if customers value the differences enough to pay more to the firm that more closely serves their particular needs. As such, the success of these strategies depends on how much a consumer values variety. To be clear, we focuse on measuring firm behavior affiliated with providing variety and not on measuring user valuation of the variety once it is provided. 
Table 1b: Sample of Differentiation Strategies

\begin{tabular}{|l|l|}
\hline $\begin{array}{l}\text { Source of } \\
\text { Differentiation }\end{array}$ & Description \\
\hline Build out & $\begin{array}{l}\text { Facilities may be built out to enhance reliability; to ease servicing } \\
\text { big clients in specific locales; or to customize service to specific } \\
\text { users needs, such as large data transfers. }\end{array}$ \\
\hline $\begin{array}{l}\text { Geographic } \\
\text { coverage }\end{array}$ & $\begin{array}{l}\text { Scope of offerings may be tailored to multi-establishment users or to } \\
\text { users in many different locations. }\end{array}$ \\
\hline Vision of services & $\begin{array}{l}\text { Services are tailored to bring value from new technology of } \\
\text { combination of services (e.g., Cable television, Internet, DSL). }\end{array}$ \\
\hline Service quality & $\begin{array}{l}\text { The CLEC provides superior after-sale services or related services } \\
\text { in network maintenance activities, such as single contracts for } \\
\text { network emergencies or contracts for bundles of voice and nonvoice } \\
\text { services. }\end{array}$ \\
\hline Non-price norms & $\begin{array}{l}\text { The CLEC may employ receptionists who smile, repair people who } \\
\text { hustle, and accounts receivable employees who quickly correct } \\
\text { billing errors. }\end{array}$ \\
\hline
\end{tabular}

In Table 1b, we provide examples of types of customer-targeted differentiation strategies commonly discussed in the trade press during the late 1990s (see NPRG 2000 for a summary). To be sure, no matter how it is deployed, every CLEC offers telephone and related data carrier services. In spite of these fundamental commonalities, CLECs and industry analysts cite particular features of firms that produce value for certain end users. The data in Table $1 \mathrm{~b}$ suggest ways that CLEC services might be tailored to customer needs.

We are not interested in evaluating the veracity of the detailed service claims made by any CLEC in any particular city. Our concern here is whether the firms' entry behavior suggests that the asserted differences affect subsequent competition. The ability to successfully differentiate may increase the likelihood of CLEC entry, since a targeted firm would earn higher revenues serving the same number of customers. As a result, two differentiated firms may be willing to enter a market that would not be attractive to two identical firms.

The differentiation between business and residential customers illustrates this general principle. By 1999, the CLEC industry had expanded beyond solely voice into services affiliated with carrying data traffic. These latter services were potentially valuable to business customers, but of more limited value to residential customers. The 
CLECs could vary their portfolio of services to target these distinct sets of customers. To the extent that these chosen services generate higher willingness to pay from their targeted customers, the differentiated CLECs would earn higher revenues. This in turn could affect market structure - there might be enough demand to support one CLEC aimed at business users and another aimed at residential users in a small city that could not support two identical CLECs that do not target a particular set of customers.

Product heterogeneity also opens questions about the asymmetric influence of demand, costs, and the regulatory setting on differentiated CLECs. For example, different components of total market size may provide demand to one type of CLEC over another. Depending on their preferences, regulatory agencies might pass rules that result in lower costs or better opportunities for some CLEC types. ${ }^{6}$

\section{II.iii Inferences about Competition and Entry in a Period of Growing Demand}

We will infer the importance of differentiation from entry behavior that is consistent with it. To be sure, entry behavior is also sensitive to industry-wide perceptions about the current state of demand and expectations for the near future by contemporary actors. A few years after the millennium it became apparent that some CLECs had been "optimistic." More precisely, some CLECs did not realize revenues sufficient to cover the debts incurred in building their facilities and marketing their new services. The trade press dates the beginning of the decline of optimism at the spring of 2000, when financial support for dot.coms collapsed. It reached a nadir in fall 2001, after the September 11 terrorist attack shook business confidence in long-term investments. This low continued as the WorldCom financial scandal became publicized in the spring of 2002. Consequently, some CLECs curtailed expansion plans they announced in 1999 and previous years. Others left the market altogether.

\footnotetext{
${ }^{6}$ Regulatory agencies could use the introduction of competition to undo cross-subsidies, thereby encouraging new entrants to price underneath regulated (i.e., artificially) high prices to business customers (e.g., Rosston and Wimmer 2001). Such a regulatory environment would comparatively favor CLECs with a business focus, but not necessarily those with a residential focus. As another example, the passage of the 1996 Telecommunications Act altered the regulatory framework for dispute resolution between ILEC and CLEC, particularly by shifting responsibility for documenting ILEC competitive (mis)behavior to state regulators. It is an open question as to what firm this change comparatively favored - a local firm having business or political connections with the state regulator or national firms with larger in-house legal experience in regulatory proceedings.
} 
This plays a role in our analysis of 1999 in some ways and not in others. We operate with the premise that expectations about demand growth beyond 1999 were optimistic, but only in retrospect were they too optimistic. We presume that contemporary actors made entry decisions on the basis of the best available information, and we ask whether their behavior is consistent with the importance of scale-exceeding efficiencies or customer-targeted differentiation. We acknowledge that deviations from a common perception about the anticipated growth of the level of demand could alter the number of CLECs a city can support. Conceivably, realized deviations from a common perception about the anticipated success of CLECs that pursue particular forms of differentiation could influence the CLEC types that survive in any market structure. In the empirical work, we assume and condition on the presence of such common perceptions. Note that, in particular, perceptions cannot be systematically different across areas of the country, since our identification strategy is based on cross-sectional differences in market structure. Our framework will focus on measuring the incentive to differentiate from a rival in the same location, as perceived at the time.

\section{Data}

Our modeling approach uses three types of information:

1. We require cross-sectional information about CLEC entry. A census of CLEC firms operating in cities across the United States come from the 1999 CLEC Report, provided by NPRG (2000).

2. We also require cross-sectional information about the economic conditions at specific localities. Information about the economic conditions in cities comes from the most recent U.S. Census data.

3. Finally, we require cross-sectional information about the regulatory environment of specific localities. Information about the regulatory environment comes from Abel and Clements' (2001) study.

\section{III.i Sample Construction}

We analyze product differentiation and competition among CLECs by studying the structure of a cross-section of markets. We attempt to distinguish between as many 
different local markets as we can, while taking care to define markets in such a way that the set of firms in the market all compete with each other (at least to some extent) and that no firms outside the defined market are competitors.

For CLECs, the most appropriate geographic market definition is at the level of individual cities. Although jurisdictional boundaries for cities do not always correspond with economic market boundaries in many industries, cities best approximate markets for CLECs. The services CLECs provide are inherently locally focused - the firm must establish a presence in a city in order to connect customers or businesses residing there. This makes most small and medium-sized cities geographically distinct market areas, even when they are suburbs in large metropolitan areas. We avoid the potential concern of distinct submarkets within cities because we do not include larger cities in our analysis. This difficulty is most serious in places such as Los Angeles or New York City. ${ }^{7}$

Similarly, the total size of the sample involved some trade-offs. We constructed a sample of every city in the United States with at least one actual CLEC entrant as of 1999, while dropping a few cities due to for incomplete information. We also wanted to include cities that are candidates for entry but that have not yet experienced it, because it helps identify the margin between any entry and none. For this purpose, we also included every city in the Untied States in which any CLEC expressed any announced plan to enter, even when these plans were several years in the future. This approach yielded over 260 cities with no actual entrants as of 1999. Also, this provided us with a convenient stopping rule, since there is little statistical benefit from including each of the thousands of small isolated cities in the United States with little economic base for supporting CLEC entry. ${ }^{8}$

\footnotetext{
${ }^{7}$ Our data set reflects this issue directly; for example, some CLECs reported operating in "New York City" while others said they offered services in "Manhattan." From these descriptions, it was impossible to discern whether the firms were competitors. Cities with potentially overlapping submarkets were removed from the final data set.

${ }^{8}$ This does, however, preclude us from estimating a threshold between cities where entry is at least planned and those with no CLEC activity at all. This threshold may also be of some policy interest, but is beyond the scope of this paper.
} 
In Table 2, we summarize the firm counts in the 718 cities where at least one CLEC was operating, or was planning to begin operations, as of 1999. The number in each box in Table 2 indicates the number of cities that have the corresponding number of operating and planned CLECs. Of the 718 cities, all but 260 had at least one firm operating as of 1999. A total of 261 cities had just one firm operating, with the numbers getting smaller through ten or more firms operating. In the planned category, no new CLECs were planning to enter in 250 cities, while 316 had one planned entrant (most of these were among the 260 cities with no operating CLECs). Again, the number of cities with CLECs in the planned category decreases rapidly across the table. There is considerable planned entry in markets of all sizes; however, the markets with the largest number of operating firms also have the largest number of planned entrants.

Table 2: Histogram of Cities: Number of Operating and Planned CLECs in the Market

\begin{tabular}{|c|c|c|c|c|c|c|c|c|c|}
\hline & \multicolumn{9}{|c|}{ Planned } \\
\hline Operating & $\boldsymbol{0}$ & $\mathbf{1}$ & $\mathbf{2}$ & $\mathbf{3}$ & $\mathbf{4}$ & $\mathbf{5}$ & $\mathbf{6 - 9}$ & $\mathbf{1 0}+$ & Total \\
\hline $\boldsymbol{0}$ & - & 238 & 17 & 4 & 1 & 0 & 0 & 0 & 260 \\
\hline $\boldsymbol{1}$ & 203 & 38 & 14 & 5 & 0 & 0 & 0 & 0 & 261 \\
\hline $\mathbf{2}$ & 36 & 21 & 13 & 5 & 2 & 1 & 0 & 0 & 78 \\
\hline $\mathbf{3}$ & 6 & 9 & 12 & 4 & 2 & 0 & 2 & 0 & 35 \\
\hline $\mathbf{4}$ & 4 & 6 & 1 & 2 & 1 & 5 & 2 & 1 & 22 \\
\hline $\mathbf{5}$ & 1 & 1 & 1 & 1 & 2 & 0 & 4 & 1 & 11 \\
\hline $\mathbf{6 - 9}$ & 0 & 3 & 0 & 1 & 5 & 5 & 10 & 4 & 28 \\
\hline $\mathbf{1 0}+$ & 0 & 0 & 1 & 0 & 0 & 1 & 10 & 11 & 23 \\
\hline Total & 250 & 316 & 59 & 22 & 13 & 12 & 29 & 17 & 718 \\
\hline
\end{tabular}

\section{III.iii Firm Characteristics and the Endogenous Variable}

We classify firms into discrete categories on the basis of their business strategies and product offerings. ${ }^{9}$ First, CLECs vary in the geographic extent of their operations. Some

\footnotetext{
${ }^{9}$ Those analysts who do acknowledge CLEC differentiation draw these distinctions similarly. Crandall and
} 
CLECs have ambitions to be providers of telecommunication services in cities across the country, whereas others focus on a more limited geographic region. Local firms may better respond to the needs of consumers in particular markets, while national CLECs may have scale economies in building reputation and can potentially offer consumers located in multiple cities a common telecommunications vendor. The NPRG (2000) data lists the complete set of cities into which each CLEC has entered. We labeled CLECs as "local/regional" if they operate completely within one city or a small number of contiguous states. Those operating in cities from multiple regions of the country are labeled "national."

In Table 3, we present the breakdown between national CLECs and the local/regional firms in each of the individual markets in the data set (note that this table only includes the cities with at least one operating firm). Here we see that the cities with fewer operating CLECs typically contain predominately local/regional firms - for example, among the markets with one firm operating, that firm is a local/regional CLEC in nearly $80 \%$ of the cases. As the total number of firms increases, however, there is a greater tendency for cities to be differentiated, with the same or close number of each type of firms as opposed to all or almost all of one type. Nearly half of the two-CLEC markets have one local/regional firm and one national firm. This general pattern holds in markets with 3 or 4 CLECs as well. Over all, this table strongly hints at the presence of differentiated entry.

From the NPRG (2000) data, we also observe that individual CLECs differentiated themselves by tailoring their service offerings to appeal to particular groups of customers. The most distinct division was between business and residential customers. Although every CLEC provided service to some business customers, we could distinguish between those that also targeted residential users and those that focused on business consumers only. Table 4 displays our markets for CLECs broken down between these two customer focus categories. Again, we observe markets that are differentiated.

Sidek (2001), for example, emphasize the differences between a focus on business and residential customers, as do we. Crandall (2001) also emphasizes the difference between building facilities and relying on rental of unbundled network elements; however, we were not able to obtain comprehensive data on these strategies and do not analyze them here. 
Of the 78 two-CLEC cities, 58 have one firm that targets its offerings to businesses and one firm that also serves residential customers. These raw data strongly suggest some underlying pattern of entry in which differentiation is optimal - if the business-only and some-residential CLECs were equally likely to enter the market under all circumstances, we would expect as many as 52 of 78 markets to have exactly one firm of each type only $0.1 \%$ of the time. These patterns continue in markets with a larger total number of CLECs as well.

Table 3: Number of National and Local/Regional CLECs per City

\begin{tabular}{|c|c|c|c|c|c|}
\hline & \multicolumn{5}{|c|}{ National CLECs } \\
\hline $\begin{array}{c}\text { Local/Regiona } \\
\boldsymbol{l} \text { CLECs }\end{array}$ & $\boldsymbol{0}$ & $\boldsymbol{1}$ & $\boldsymbol{2}$ & $\mathbf{3}$ & $4+$ \\
\hline $\boldsymbol{0}$ & N/A & 69 & 5 & 3 & 1 \\
\hline $\boldsymbol{1}$ & 214 & 35 & 10 & 3 & 2 \\
\hline $\boldsymbol{2}$ & 38 & 11 & 9 & 3 & 1 \\
\hline 3 & 12 & 6 & 4 & 1 & 5 \\
\hline $4+$ & 4 & 2 & 8 & 6 & 2 \\
\hline
\end{tabular}

Table 4: Number of Business Only and Some Residential CLECs per City

\begin{tabular}{|c|c|c|c|c|c|}
\hline & \multicolumn{5}{|c|}{ Business Only } \\
\hline $\begin{array}{c}\text { Some } \\
\text { Residential }\end{array}$ & $\boldsymbol{0}$ & $\boldsymbol{1}$ & $\boldsymbol{2}$ & $\mathbf{3}$ & $\mathbf{4 +}$ \\
\hline $\boldsymbol{0}$ & N/A & 144 & 15 & 5 & 6 \\
\hline $\boldsymbol{1}$ & 139 & 52 & 21 & 8 & 7 \\
\hline $\boldsymbol{2}$ & 11 & 8 & 8 & 4 & 7 \\
\hline 3 & 2 & 2 & 1 & 2 & 4 \\
\hline $4+$ & 1 & 1 & 1 & 3 & 3 \\
\hline
\end{tabular}

Before leaving our discussion of product differentiation and the definition of the dependent variable, we investigate the relationship between the two dimensions of 
heterogeneity. In Table 5, we summarize the pair of characteristics for the individual CLECs operating in 1999. We found no apparent correlation between the customer focus and the geographic scope of individual CLECs. In other words, a national CLEC was as likely to maintain a business versus a residential focus as was a local/regional CLEC. Had we found a correlation, we would have tried to assess both aspects of product differentiation simultaneously. Since we do not, we analyze each axis of differentiation separately. ${ }^{10}$

Table 5: Number of Business Only and Some Residential CLECs

\begin{tabular}{|c|c|c|c|}
\hline & Business only & $\begin{array}{c}\text { Some } \\
\text { residential }\end{array}$ & Total \\
\hline Local/Regional & 31 & 44 & 75 \\
\hline National & 16 & 13 & 29 \\
\hline Total & 47 & 57 & 104 \\
\hline
\end{tabular}

The tables in this section show how CLECs with different classifications sort themselves into markets of various sizes. The basic empirical fact in these tables portend our main findings: The CLECs acted as if differentiating their services provided additional profitability and facilitated entry into local markets in the late 1990s. A pair of CLECs in the same market most likely was distinguished by differentiated service offerings.

\section{III.iii Economic Data about Localities}

Cities will differ in their ability to generate the necessary demand to make CLEC entry attractive. To account for these differences, we collected demographic data from each city. Market "size" was the most important of these characteristics - here, we are interested in both the resident population and measures of business activity, since CLEC services are often particularly valuable to business customers. Population is each city's population and per capita income represents the average income of the city's residents.

\footnotetext{
${ }^{10}$ Even if we found a correlation, it is not clear that we even have sufficient data to identify complementarity or substitutability. Athey and Stern (1998) detail the strict data requirements necessary to identify complementarities in a cross-sectional study such as this. Even if complementarities were assumed to be zero, a very large data set would be required in the case in which competing firms could choose among four potential options (one of each type from two categories) simultaneously.
} 
The variable payroll measures the annual payroll of workers employed in the city; as such it combines both the overall level of business activity and the wages earned by workers. Summary statistics for all of the explanatory variables are included in Table 6 .

As described above, we hypothesize that CLECs are able to share costs (such as marketing, administration, and initial costs associated with interconnection) among nearby cities (even if separate facilities are built). It is possible, therefore, that a small city within a larger Metropolitan statistical area (MSA) may be less expensive for a firm to serve than a more isolated city of equal size. We include the dummy variable city in a top-10 MSA to represent those cities that are within the boundaries of one of the ten largest urban areas in the United States. We confirm that the dummies were plausibly related to our proposed interpretation of scope economies by inspecting the CLECs that entered the city in a top-10 MSA. Of the 381 CLEC observations in such cities, 333 of them also operated in the MSAs corresponding central city. ${ }^{11}$

We also examine cost differences across markets that relate to the local regulatory environment. Abel and Clements' (2001) study provides us with a time-series of regulatory rules that states have used on ILECs under their jurisdiction. ${ }^{12}$ For each state/ILEC combination, we counted the number of years (of the previous sixteen) that either a rate freeze or price cap had been imposed. Our summary measure of regulatory stringency is a collapsed version of this year count - regulatory stringency equals 0 in areas where these alternatives had never been used, 1 if they had been tried for between one and four years, and 2 if a freeze or cap was in place for more than five years. We hypothesize that a higher value of regulatory stringency indicates a regulatory environment that would be friendlier (i.e., be lower in cost) to an entering CLEC.

\footnotetext{
${ }^{11}$ We explored several other demographic variables as well. Because the facilities-based CLECs must make capital investments in equipment to link their customers, cities with more geographically concentrated residential neighborhoods and business centers may provide CLECs with customers that are less expensive to serve. However, the density measures that we calculated (both residential and for businesses) did not provide additional explanatory power. It may be that density differences affect where CLECs operate within cities but not entry decisions across cities.

${ }^{12}$ Regulators often have different rules for each incumbent carrier within its state. These rules apply to all the areas within that state where the particular incumbent operates. Therefore, it was necessary to match each market to both its incumbent and its state regulator to determine the status of the incumbent competitor.
} 
Table 6: Explanatory Variables — Summary Statistics

\begin{tabular}{|l|c|c|c|c|c|}
\hline & & \multicolumn{2}{|c|}{ All Cities (n= 718) } & \multicolumn{2}{|c|}{$\begin{array}{c}\text { Small/Medium-Sized } \\
\text { Cities (n = 65) }\end{array}$} \\
\hline Label & Definition & Mean & $\begin{array}{c}\text { Standard } \\
\text { Deviation }\end{array}$ & Mean & $\begin{array}{c}\text { Standard } \\
\text { Deviation }\end{array}$ \\
\hline Population & City population & 137,143 & 357,890 & 85,270 & 110,244 \\
\hline Payroll & $\begin{array}{c}\text { Annual payroll of city } \\
\text { employees }\end{array}$ & $2,196,817$ & $6,544,441$ & $1,247,663$ & $1,802,501$ \\
\hline $\begin{array}{l}\text { Per Capita } \\
\text { Income }\end{array}$ & $\begin{array}{c}\text { Per capita income of } \\
\text { city residents }\end{array}$ & 14,542 & 5,376 & 14,517 & 5,518 \\
\hline $\begin{array}{l}\text { City in } \\
\text { Top-10 } \\
\text { MSA }\end{array}$ & $\begin{array}{c}\text { Dummy Variable }=1 \\
\text { if city within Top 10 } \\
\text { MSAs }\end{array}$ & 0.252 & 0.434 & 0.244 & 0.430 \\
\hline $\begin{array}{l}\text { Incumbent } \\
=\text { RBOC }\end{array}$ & $\begin{array}{c}\text { Dummy variable }=1 \text { if } \\
\text { ILEC is an RBOC }\end{array}$ & 0.726 & 0.447 & 0.720 & 0.449 \\
\hline $\begin{array}{l}\text { Regulatory } \\
\text { Stringency }\end{array}$ & $\begin{array}{c}\text { Regulatory stringency } \\
\text { variable (described in } \\
\text { text) }\end{array}$ & 1.152 & 0.687 & 1.147 & 0.687 \\
\hline $\begin{array}{l}\text { Inter- } \\
\text { connection } \\
\text { rate }\end{array}$ & $\begin{array}{c}\text { Interconnection rate } \\
\text { set by state regulatory } \\
\text { agency }\end{array}$ & 16.22 & 6.24 & 16.44 & 6.30 \\
\hline
\end{tabular}

Specific provisions in the 1996 Telecommunications Act require incumbents to provide interconnection access to CLEC competitors; however, RBOC firms that wanted to enter the market for long-distance services were precluded from doing so until regulators were satisfied that they had been sufficiently cooperative with CLECs attempting to interconnect and provide service in their local areas. Incumbents that were not RBOCs did not have this incentive to facilitate CLEC entry. Following on the results of Mini (2001), we included an RBOC dummy variable to control for the differing incumbent incentives across the markets in the data set. In addition, local regulators prescribed the costs that CLECs were required to pay ILECs for interconnection. These rates vary by and within each state; typically they were set lower in more densely populated areas. Using data from Gregg's (2002) and Rosston and Wimmer's (2001) studies, we created the variable interconnection rate to capture the cross-sectional 
differences in interconnection costs for CLECs entering across cities in the data set. ${ }^{13}$ While entry costs for a CLEC depend on regulated rates for a variety of unbundled elements, most of these rates are correlated with each other in the cross section. Hence, one variable alone captures the biggest differences between locations. ${ }^{14}$

\section{Empirical Models of CLEC Entry}

The empirical modeling approach that we utilize fits into the series of "multiple-agent qualitative-response" frameworks introduced into industrial organization literature to evaluate entry strategies and market competition. Using a cross-section of markets as data, the econometrician infers the economic factors that contribute to the generation of the observed market structure. Firms' strategies can be represented by discrete decisions (e.g., enter/do not enter a particular market) that are made by evaluating the potential alternatives. Estimation is complicated by the fact that the decisions of competing firms may affect potential alternatives - for example, entry may be less attractive if other firms also have entered the market. A game-theoretic behavioral model is used to infer the factors influencing individual firm decisions from an observed market structure outcome, which is determined by the choices made by interacting agents.

\section{IV.i Entry Models Assuming Homogeneity}

Much of the industrial organization literature, as well as previous empirical papers on CLEC entry, have estimated straightforward limited dependent variable models of market structure. The simplest of these analyses are probits that predict the likelihood of entry across markets; other studies estimate ordered probits with the number of firms as the dependent variable. The market factors that determine entry are inferred from a profit function that underlies the entry decision, such as

\footnotetext{
${ }^{13}$ Specifically, we use the unbundled network element (UNE) loop rate to proxy for the cost of interconnection, as this represents the bulk of network connection costs. The CLECs may differ in the particular UNEs that they need from the incumbent; however, these differences are orthogonal to the product types that we are examining here.

${ }^{14}$ We also explored specifications for cross-sectional differences in the margins between regulated telephony prices and regulated costs, as estimated by Gregg (2002). We found that the estimate of margins and the estimate of interconnection rate were highly correlated, so we only included one in the final specification.
} 


$$
\pi_{m}=X_{m} \beta-Z_{m} \gamma-N_{m} \theta+\varepsilon_{m}
$$

where $m$ denotes the geographic market in question. Markets may differ in the crosssection in $X$-characteristics affecting demand for the firms' products or in $Z$ characteristics that affect the firms' market-specific costs. $N_{m}$ represents the number of firms that have entered the market; $\theta$ indicates the extent to which additional market participants makes entry less attractive. The $\varepsilon_{m}$ term represents the components of firm profits that are unobserved to the econometrician.

The parameters of this function can be estimated using a cross-section of market structure observations. For example, we may observe two firms operating in market $m$. This implies the following inequalities in market $m$ :

$$
\begin{aligned}
& \pi_{m}=X_{m} \beta-Z_{m} \gamma-(2) * \theta+\varepsilon_{m}>0 \\
& \pi_{m}=X_{m} \beta-Z_{m} \gamma-(3) * \theta+\varepsilon_{m}<0
\end{aligned}
$$

The parameters are determined by maximizing the likelihood that the inequalities implied by the observed market structures (assuming a distribution for the market level error term) hold.

\section{IV.ii Extensions to Heterogeneous Markets}

This approach can be extended to analyze firms in heterogeneous markets as well. Suppose that each market could have firms of two types, label them A and B. Now, market structure is represented by an ordered pair $\left(\mathrm{N}_{\mathrm{A}}, \mathrm{N}_{\mathrm{B}}\right)$ indicating the number of observed firms of each type. In addition, there will be type-specific profit functions for these firms:

$$
\pi_{T m}=X_{m} \beta_{T}-Z_{m} \gamma_{T}-N_{T m} \theta_{T}-N_{-T m} \theta_{-T}+\varepsilon_{T m},
$$

where the cost and demand effects can be type-specific and where we can allow the effects of competitors to vary on the basis of whether they offer the same or different product types. $N_{T m}$ indicates the number of same-type firms in the market, and $N_{-T m}$ is the number of firms of the other type. Therefore, the difference between the estimated $\theta_{T}$ and $\theta_{-T}$ parameters captures the extent to which product differentiation may limit the effects of additional competitors on firm entry of each type. The unobserved part of profits, $\varepsilon_{T m}$, is assumed to be different for each product type at a given market. 
With multiple product types, the set of inequalities that corresponds to each market outcome is necessarily more complex. A market observed with a structure of $(\mathrm{A}, \mathrm{B})$ implies that the following inequalities hold:

$$
\begin{array}{lll}
\pi_{A}(A, B)>0 & \pi_{A}(A+1, B)<0 & \pi_{A}(A, B)>\pi_{B}(A-1, B+1) \\
\pi_{B}(A, B)>0 & \pi_{B}(A, B+1)<0 & \pi_{B}(A, B)>\pi_{A}(A+1, B-1)
\end{array}
$$

Again, we estimate the parameters by maximizing the likelihood that the inequalities corresponding to each observed outcome hold across the markets in the data set. (For further details, see Mazzeo's 2002 study.)

\section{IV.iii Identification and Testing}

Using cross-sectional differences in observed market structure, thisthe following approach identifies parameters describing the attractiveness of entry.. It is best suited for analysis of small and medium-sized cities, because with many firms the marginal effect of additional competitors is likely to be very small. Consequently, we focus on measuring the key differences between cities that may become substantially more competitive with additional entry - places such as Buffalo, Little Rock, and Fresno. We believe that for policy purposes, these markets are most interesting; after all, larger cities will likely be competitive under almost any set of local and national regulations for CLECs (Woroch 2001).

Intuitively speaking, identification of differentiation comes from comparing otherwise similar markets with different structures or, conversely, different markets with otherwise similar structures. Two markets with the same number of firms may not be otherwise equal - if the firms are more heterogeneous in one market, their differentiation may accompany a smaller population. A particular market with insufficient demand to support two homogeneous firms may have enough demand for two firms that differentiate. Recall that we conceptualize the function that underlies the market structure observations as a profit function, even though firms may have uncertainty about whether variable profits will exceed their costs of entry. To the extent that markets are not in equilibrium at the times of our analysis, we are more precisely measuring firms' expectations about profitability and how these expectations are affected by competition and differentiation. We assume common perceptions about profitability 
and assume that any potential deviation between perceptions and subsequent realizations are not correlated across markets or product types.

Finally, it is appropriate to think of our classification as a maintained assumption. The framework does not measure whether our classification of differentiation corresponds with success at executing the strategies identified in Table 1b, nor do we measure how highly users valued particular types of CLECs. Our null hypothesis is that firms enter without regard to the product type of their competitors, using the business versus residential and local/regional versus national classifications from the previous section. If we fail to reject the null hypothesis, we do so either because firms do not differentiate from their local competitors or because we have inappropriately classified the dimensions in which they differentiate. Such a failure to reject could arise, for example, if differentiation between resale and facilities-based CLECs is actually what matters and it is orthogonal to the classifications we do analyze. If we reject the null hypothesis, then we accept the alternative hypothesis that CLECs enter local markets in such a way as to differentiate along the dimensions we classify.

\section{Empirical Results}

To provide a comparison with earlier work, we begin our empirical analysis by estimating probits predicting CLEC entry and ordered probits whose dependent variables are the numbers of operating CLECs. The explanatory variables highlight the foundations of scale-exceeding efficiencies - local market demand, geographic scope, and regulatory environment. Unlike the previous analysis of CLEC entry, however, we examine city-level markets. ${ }^{15}$ The finely grained data should yield insight about the precise relationship between local economic factors and entry levels. These estimates also provide a useful benchmark against our later estimates that account for differentiation.

Tables 7 and 8 present the results from two estimations — in the first the dependent variable is AOPERATE, a dummy variable that equals one if any CLECs are currently

\footnotetext{
${ }^{15}$ As was previously mentioned, prior studies of CLEC entry used individual LATAs as the unit of observation. Each LATA may contain several individual city markets, as defined in our data set.
} 
operating within that market. ${ }^{16}$ We also present an ordered probit estimation, where the dependent variable OPERATE is a count of the number of CLECs doing business in the market.

Table 7: Probit of CLEC Entry — Dependent Variable = AOPERATE

\begin{tabular}{|l|c|c|c|c|c|c|}
\hline & \multicolumn{2}{|c|}{ Small/Medium Sized Cities } & \multicolumn{3}{c|}{ All Cities } \\
\hline & Coefficient & $\begin{array}{c}\text { Standard } \\
\text { Error }\end{array}$ & $Z$ & Coefficient & $\begin{array}{c}\text { Standard } \\
\text { Error }\end{array}$ & $Z$ \\
\hline Constant & -0.635 & 0.316 & -2.01 & -0.627 & 0.316 & -1.98 \\
\hline Population & $3.29 \mathrm{e}-6$ & $1.48 \mathrm{e}-6$ & 2.23 & $3.31 \mathrm{e}-6$ & $1.48 \mathrm{e}-6$ & 2.24 \\
\hline Payroll & $3.17 \mathrm{e}-7$ & $1.13 \mathrm{e}-7$ & 2.81 & $3.23 \mathrm{e}-7$ & $1.13 \mathrm{e}-7$ & 2.85 \\
\hline $\begin{array}{l}\text { Per Capita } \\
\text { Income }\end{array}$ & $2.56 \mathrm{e}-5$ & $1.54 \mathrm{e}-5$ & 1.67 & $2.55 \mathrm{e}-5$ & $1.54 \mathrm{e}-5$ & 1.66 \\
\hline $\begin{array}{l}\text { City in Top- } \\
\text { 10 MSA }\end{array}$ & 0.254 & 0.168 & 1.51 & 0.250 & 0.168 & 1.48 \\
\hline $\begin{array}{l}\text { Incumbent } \\
=\text { RBOC }\end{array}$ & 0.288 & 0.126 & 2.30 & 0.283 & 0.125 & 2.26 \\
\hline $\begin{array}{l}\text { Regulatory } \\
\text { Stringency }\end{array}$ & 0.302 & 0.087 & 3.47 & 0.300 & 0.087 & 3.44 \\
\hline $\begin{array}{l}\text { Inter- } \\
\text { connection } \\
\text { rate }\end{array}$ & -0.033 & 0.010 & -3.43 & -0.033 & 0.010 & -3.44 \\
\hline
\end{tabular}

Note: For an easier comparison with the following analysis, cities with seven or more operating CLECs were excluded from the left-hand panel. There are 675 observations, with 718 in the full data set of cities.

All three demographic variables are positively correlated with CLEC entry, with population and payroll being particularly significant. Entry is positively affected by both the demand generated by consumers that live in the city and by businesses that operate within the city. Interestingly, these two variables have somewhat greater predictive value in the OPERATE ordered probit. This suggests that AOPERATE is a relatively coarse measure, at least as far as approximating CLEC demand. We also see that the city in a top-10 MSA dummy is positive in both estimations, but more significant in the AOPERATE probit. There appears to be some (albeit weak) evidence of scope

\footnotetext{
${ }^{16}$ Our set of markets that have zero operating firms, by construction, have at least one CLEC that is planning to enter. This method of sample construction results in a wide array of cities - many with populations under 50,000 and some in otherwise low-density rural areas. Still, this is a fully random sample in the exogenous variables, so this method will bias the coefficients in Table 7 slightly downward, as compared to a sample that also includes even tinier cities in which no CLECs had plans to enter.
} 
economies, since CLEC entry is more common in small and medium-sized cities within larger MSAs, all else being equal.

The regulatory variables $R B O C$, regulatory stringency and interconnection rate are all significant and have the expected sign. It appears easier for CLECs to enter in areas where RBOCs are the incumbent local service providers; this is consistent with the prediction that the regulatory incentives would make areas with RBOCs less hostile toward potential entrants. ${ }^{17}$ The CLECs are also more common in areas where the regulators had experimented away from traditional rate-of-return systems for longer periods of time and where interconnection rates are set lower. These results suggest that the regulatory environment can have both a long- and short-term effect on markets.

Table 8: Ordered Probit of CLEC Firm Counts - Dependent Variable $=$ OPERATE

\begin{tabular}{|l|c|c|c|c|c|c|}
\hline & \multicolumn{2}{|c|}{ Small/Medium Sized Cities } & \multicolumn{3}{c|}{ All Cities } \\
\hline & Coefficient & $\begin{array}{c}\text { Standard } \\
\text { Error }\end{array}$ & $Z$ & Coefficient & $\begin{array}{c}\text { Standard } \\
\text { Error }\end{array}$ & $Z$ \\
\hline Population & $4.40 \mathrm{e}-6$ & $7.75 \mathrm{e}-7$ & 5.68 & $1.65 \mathrm{e}-6$ & $5.23 \mathrm{e}-7$ & 3.15 \\
\hline Payroll & $1.55 \mathrm{e}-7$ & $4.79 \mathrm{e}-8$ & 3.24 & $2.08 \mathrm{e}-7$ & $3.29 \mathrm{e}-8$ & 6.32 \\
\hline $\begin{array}{l}\text { Per Capita } \\
\text { Income }\end{array}$ & $1.70 \mathrm{e}-5$ & $9.55 \mathrm{e}-6$ & 1.78 & $1.40 \mathrm{e}-5$ & $9.22 \mathrm{e}-6$ & 1.52 \\
\hline $\begin{array}{l}\text { City in Top- } \\
\text { 10 MSA }\end{array}$ & 0.138 & 0.117 & 1.18 & 0.059 & 0.112 & 0.53 \\
\hline $\begin{array}{l}\text { Incumbent } \\
\text { RBOC }\end{array}$ & 0.206 & 0.102 & 2.02 & 0.253 & 0.098 & 2.58 \\
\hline $\begin{array}{l}\text { Regulatory } \\
\text { Stringency }\end{array}$ & 0.160 & 0.068 & 2.34 & 0.172 & 0.066 & 2.62 \\
\hline $\begin{array}{l}\text { Inter- } \\
\text { connection } \\
\text { rate }\end{array}$ & -0.029 & 0.008 & -3.56 & -0.035 & 0.008 & -4.35 \\
\hline
\end{tabular}

Note: For an easier comparison with the following analysis, cities with seven or more operating CLECs were excluded from the left-hand panel. There are 675 observations, with 718 in the full data set of cities.

\footnotetext{
${ }^{17}$ We also tried dummy variables for individual RBOCs; however, when averaged over all the cities where the incumbents operate, the effect of individual RBOCs could not be distinguished from their aggregate impact relative to non-RBOC incumbents.
} 


\section{V.i Product Heterogeneity Estimates}

In the heterogeneous products analysis, we allow for up to three firms of each product type in the market - therefore, the endogenous market structure variable can take on one of sixteen possible values. The information in Tables 3 and 4 capture the variation in the dependent variable across all the markets in the data set. ${ }^{18}$ For each firm type and market configuration, a set of dummy variables is defined, and the corresponding $\theta$-parameters represent the incremental effects of additional competitors on the profits of firms in the market. For example, in the comparison between the business-only and some-residential CLECs, we have ${ }^{19}$

$\theta_{R R I}=$ effect of first some-residential competitor on some-residential CLECs,

$\theta_{R R 2}=$ effect of second some-residential competitor on some-residential CLECs,

$\theta_{R B}=$ effect of business-only competitors on some-residential CLECs,

$\theta_{B B I}=$ effect of first business-only competitor on business-only CLECs,

$\theta_{B B 2}=$ effect of second business-only competitor on business-only CLECs, and

$\theta_{B R}=$ effect of some-residential competitors on business-only CLECs.

The estimated parameters can be used to evaluate the null hypothesis of homogeneous competition. A strict test of that property is $\left|\theta_{R R I}\right|=\left|\theta_{R B}\right|$ and $\left|\theta_{B B I}\right|=\left|\theta_{B R}\right|$. We can reject the null in favor of a model of differentiated competition if we find $\left|\theta_{R R I}\right|>\left|\theta_{R B}\right|$ and $\left|\theta_{B B I}\right|$ $>\left|\theta_{B R}\right|$. There are similar tests for homogeneity and differentiation with respect to the national versus local/regional classifications. Notice that in the absence of within-type heterogeneity, we would expect to find $\left|\theta_{R R I}\right|>\left|\theta_{R R 2}\right|$ and $\left|\theta_{B B I}\right|>\left|\theta_{B B 2}\right|$.

As in the probit estimations, the appropriate $X$-variables to include are either correlated with CLEC demand or entry costs in each market. The specification also allows the effects associated with the $X$-variables to vary by product type. To ease estimation, the data for the $X$-variables are transformed to the log of the actual value for

\footnotetext{
${ }^{18}$ For example, there are eleven markets whose dependent variable is $(2,1)$ in the geographical ambition model - two local operating CLECs and one national operating CLEC (cf. Table 3). Cities with more than three firms in either category are treated as if they have exactly three in that category.

${ }^{19}$ The goal is to make the specification of the competitive effects as flexible as possible, while maintaining estimation feasibility. More flexible parameterizations of these effects did not yield further economic distinctions.
} 
that market divided by the sample mean of that $X$-variable across all the markets in the data set. Consequently, a value of $X$ equal to the sample mean becomes zero, a value above the mean becomes positive and a value below the mean becomes negative. ${ }^{20}$ This also eases interpretation because it puts all variables on the same scale and allows for a quick comparison of the economic importance of competing variables. For example, we can say that differentiation is "economically important" if it is as large as the variance in other exogenous variables, such as city size and income, which are known to shape the number of overall entrants.

We present the results from the heterogeneous products analysis below, with the local/regional versus national classification in Table 9 and residential versus business classification in Table 10. In each table, the estimated parameters indicate the impact on entry of each type of CLEC depending on market conditions and the competitors they face. For example, the relative value of the constants indicates the relative baseline attractiveness of entry for each type. Table 9 indicates that, all else being equal, a national CLEC would be more likely to enter before a local/regional CLEC, since $C_{N}=$ $0.8695>C_{L}=0.4961 .^{21}$ The advantage is less clear in Table 10, where the constant for some-residential, $C_{R}=0.2466$, is only slightly greater than that for business-only, namely, $C_{B}=0.1463$.

Factoring in market conditions can change this relationship. For example, consider the payroll variable - the parameter estimate for both product types is positive, indicating that greater business activity attracts CLECs of either type. However, the estimated parameter is higher for the national CLEC than that for the local/regional CLEC. This indicates that as the payroll in a city increases, the relative attractiveness of entry for national CLECs increases as well. To illustrate how this can change market structure, suppose that in market $m$, payroll is half the sample mean, city in a top-10 $M S A, R B O C$ and regulatory stringency are set to zero, and the other $X$-variables are at

\footnotetext{
${ }^{20}$ The transformation is done solely to facilitate estimation of the model. The estimation routine converges more easily if the ranges of the independent variables are similar to each other.

${ }^{21}$ All the figures presented in this section represent predicted values. The comparisons between product types assume that values of the unobservables for both types are at their mean - zero. Directly evaluating the probability that one type's entry is more likely than the other's requires the standard errors of the parameters, as well as an assumption about the variance of the errors for each type.
} 
their sample means. With no competitors, operating a local/regional CLEC is now more attractive $\left[\pi_{L}=0.4961+(-0.693) *(0.2430)=0.328\right]$ than a national CLEC $\left[\pi_{N}=0.8695\right.$ $+(-0.693) *(0.8943)=0.250] .^{22}$ This combination helps explain why the one-CLEC markets in the data set are more skewed toward local/regional CLECs (see Table 3) small cities have less business activity and their lower business activity offsets the relative value of the constants.

The key result in the two tables comes from the estimated competitive effects on CLEC type, as captured by the $\theta$-parameters. The estimates indicate that the effects of competitors come predominately from same-type CLECs. In Table 9, we observe that the presence of a similar competitor makes entry quite unattractive $\left(\theta_{L L 1}=-1.18 ; \theta_{N N 1}=\right.$ $-0.81)$. as compared to the presence of competitors of the other product type $\left(\theta_{L N}=-\right.$ $\left.0.00 ; \theta_{N L}=-0.06\right)$. The estimates are similar in Table 10 for the some-residential CLECs $\left(\theta_{R R 1}=-1.17 ; \theta_{R B}=-0.00\right)$, though not quite as strong for the business-only firms $\left(\theta_{B B 1}=\right.$ $\left.-1.24 ; \theta_{B R}=-1.12\right)$. This comparison accords with economic intuition. Recall that the classification scheme here is between CLECs that are targeted just to businesses (business-only) and CLECs that serve residential customers as well as businesses (someresidential). By construction, firms are targeting overlapping customer bases. Hence, it is not surprising that some-residential CLECs have a measurable competitive effect on the business-only firms. ${ }^{23}$ In contrast, national firms target customers that differ from the local firms, so each type of firm pursues strategies more distinct from each other.

\footnotetext{
${ }^{22}$ With payroll half the sample mean, the parameter estimate for income is multiplied by $\ln (0.5)=(-0.693)$, to compute the prediction. The transformed value of an $X$-variable at its sample mean is zero; therefore, the other variables do not contribute to the prediction.

${ }^{23}$ The customer overlap is not symmetric, as the presence of business-only competitors does not have an effect on the some-residential CLECs. This may be because a business-only CLEC is not a relevant alternative for part of the some-residential CLEC's customer base, whereas the some-residential CLEC would potentially be an alternative for all of a business-only CLEC's customers.
} 
Table 9 - Profit Function Estimates from Two-Type Product Choice Model "National" and "Local/Regional" CLECs

\begin{tabular}{|c|c|c|c|}
\hline \multicolumn{2}{|c|}{ 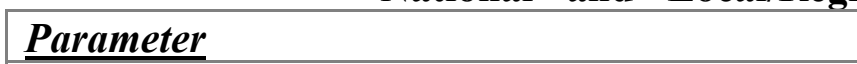 } & Estimate & Standard Error \\
\hline \multicolumn{4}{|l|}{$\overline{\text { Effect on Local/Regional CLECS }}$} \\
\hline Constant & $C_{L}$ & $0.4961 *$ & 0.1385 \\
\hline Local/Regional Competitor \#1 & $\theta_{L L 1}$ & $-1.1809 *$ & 0.1010 \\
\hline Local/Regional Competitor \#2 & $\theta_{L L 2}$ & $-0.8523 *$ & 0.1141 \\
\hline \# of National Competitors & $\theta_{L N}$ & -0.0001 & 0.0043 \\
\hline Population & $\beta_{L-P O P}$ & $0.0518^{*}$ & 0.0110 \\
\hline Payroll & $\beta_{L-P A Y}$ & $0.2430 *$ & 0.0456 \\
\hline Per-Capita Income & $\beta_{L-I N C}$ & -0.3306 & 0.2802 \\
\hline City in a Top-10 MSA & $\beta_{L-M S A}$ & -0.3192 & 0.2228 \\
\hline Incumbent $=$ RBOC & $\beta_{L-R B O C}$ & $-0.3340 *$ & 0.1489 \\
\hline Regulatory Stringency & $\beta_{L-R E G E X P}$ & 0.0139 & 0.1127 \\
\hline Interconnection Rate & $\beta_{L-L O O P R A T E}$ & $-0.9084 *$ & 0.3208 \\
\hline \multicolumn{4}{|l|}{ Effect on National CLECS } \\
\hline Constant & $C_{N}$ & $0.8695^{*}$ & 0.1910 \\
\hline National Competitor \#1 & $\theta_{N N 1}$ & $-0.8062 *$ & 0.0744 \\
\hline National Competitor \#2 & $\theta_{N N 2}$ & $-0.9518 *$ & 0.0965 \\
\hline \# of Local/Regional Competitors & $\theta_{N L}$ & $-0.0642 *$ & 0.0054 \\
\hline Population & $\beta_{N-P O P}$ & $-0.0338 *$ & 0.0047 \\
\hline Payroll & $\beta_{N-P A Y}$ & $0.8943 *$ & 0.1205 \\
\hline Per Capita Income & $\beta_{N-I N C}$ & $-1.0413 *$ & 0.3416 \\
\hline City in a Top-10 MSA & $\beta_{N-M S A}$ & $0.7591^{*}$ & 0.2434 \\
\hline Incumbent $=$ RBOC & $\beta_{N-R B O C}$ & $-0.7921 *$ & 0.2000 \\
\hline Regulatory Stringency & $\beta_{N-R E G E X P}$ & $-0.6350 *$ & 0.1673 \\
\hline Interconnection Rate & $\beta_{N-L O O P R A T E}$ & -0.0618 & 0.4445 \\
\hline
\end{tabular}

* Denotes statistical significance at the $1 \%$ level.

These results provide strong evidence that CLECs enter markets in a pattern consistent with substantial returns to product differentiation - customer-targeted differentiation appears to insulate CLECs from lower margins that typically result form reduced competition in homogeneous product markets. While the differences across product types is generally quite stark, substantial heterogeneity within types likely remains. The effect of additional same-type competitors is about the same as the effect of the first same-type competitor. Remaining differences within product types is consistent with lower prospects for entrants that face competition, but not low enough to deter entry entirely. 
Table 10 - Profit Function Estimates from Two-Type Product Choice Model "Some-Residential" and "Business-Only" CLECs

\begin{tabular}{|c|c|c|c|}
\hline \multicolumn{2}{|l|}{ Parameter } & Estimate & Standard Error \\
\hline \multicolumn{4}{|l|}{ Effect on Some-Residential CLECS } \\
\hline Constant & $C_{R}$ & $0.2466^{*}$ & 0.1046 \\
\hline Some-Residential Competitor \#1 & $\theta_{R R 1}$ & $-1.1676^{*}$ & 0.1113 \\
\hline Some-Residential Competitor \#2 & $\theta_{R R 2}$ & $-1.7414^{*}$ & 0.2179 \\
\hline \# of Business-Only Competitors & $\theta_{R B}$ & -0.0000 & 0.0000 \\
\hline Population & $\beta_{R-P O P}$ & $0.0541 *$ & 0.0107 \\
\hline Payroll & $\beta_{R-P A Y}$ & $0.1794 *$ & 0.0426 \\
\hline Per Capita Income & $\beta_{R-I N C}$ & $0.7306^{*}$ & 0.2325 \\
\hline City in a Top-10 MSA & $\beta_{R-M S A}$ & $0.5561 *$ & 0.2051 \\
\hline Incumbent $=$ RBOC & $\beta_{R-R B O C}$ & -0.0003 & 0.0015 \\
\hline Regulatory Stringency & $\beta_{R-R E G E X P}$ & 0.0029 & 0.0034 \\
\hline Interconnection Rate & $\beta_{R \text {-LOOPRATE }}$ & $-0.9246^{*}$ & 0.3096 \\
\hline \multicolumn{4}{|l|}{ Effect on Business-Only CLECS } \\
\hline Constant & $C_{B}$ & $0.1463 *$ & 0.1055 \\
\hline Business-Only Competitor \#1 & $\theta_{B B 1}$ & $-1.2443 *$ & 0.0799 \\
\hline Business-Only Competitor \#2 & $\theta_{B B 2}$ & $-1.1097 *$ & 0.1125 \\
\hline \# of Some-Residential Competitors & $\theta_{B R}$ & $-1.1234^{*}$ & 0.0944 \\
\hline Population & $\beta_{B-P O P}$ & $0.0377 *$ & 0.0102 \\
\hline Payroll & $\beta_{B-P A Y}$ & $0.3606^{*}$ & 0.0625 \\
\hline Per Capita Income & $\beta_{B-I N C}$ & 0.1280 & 0.2280 \\
\hline City in a Top-10 MSA & $\beta_{B-M S A}$ & -0.1156 & 0.2230 \\
\hline Incumbent $=$ RBOC & $\beta_{B-R B O C}$ & -0.0893 & 0.0999 \\
\hline Regulatory Stringency & $\beta_{B-R E G E X P}$ & 0.0977 & 0.1080 \\
\hline Interconnection Rate & $\beta_{B-L O O P R A T E}$ & $-1.0268^{*}$ & 0.3381 \\
\hline
\end{tabular}

* Denotes statistical significance at the $1 \%$ level.

Comparing the $\beta$-parameters between the upper and lower panels of the two Tables illustrates how certain demographic variables and market conditions support one CLEC type more or less than the other. As illustration, consider the market demand hypothesis that was evaluated using the per capita income variable. The probit estimates indicates that cities with higher per capita income were more attractive for entry; the differentiated products analysis in Table 9 suggests that the some-residential CLECs find entry in such areas relatively more attractive $\left(\beta_{\mathrm{R}-\mathrm{INC}}=0.73\right)$ than the business-only CLECs $\left(\beta_{\mathrm{B}-\mathrm{INC}}=\right.$ 0.13). The effect of the regulatory environment can also differ by product type, as the 
local/regional CLECs get a positive boost in areas where the regulators have set lower interconnection rates $\left(\beta_{\mathrm{L} \text {-LOOPRATE }}=-0.91\right)$. The national CLECs appear to have entry strategies that are largely independent of local cost conditions $\left(\beta_{\mathrm{N} \text {-LOOPRATE }}=-0.06\right)$, which suggests that the incentive to build a wide geographic base of operations is more powerful that the effect of interconnection costs at individual markets.

The differences in the $\beta$ coefficients provide further evidence of the merits of modeling differentiation. All the coefficients between national and regional/local CLECs are statistically different from each other, which indicates that the two types of firms are attracted to much different locations. The same is not so for the coefficients on the estimates for business-only and some-residential CLECs. Some of the coefficients differ, principally payroll, per capita income, and city in top-10 MSA. As with our earlier findings, this indicates that business-only and some-residential firms differ from each other, but not as much. For example, the interconnection rate has the same effect on both types.

Taken together, these results strongly suggest that the differentiation strategies of CLECs up through 1999 precipitated entry in many local markets. The estimated parameters are consistent with the notion that entry was more attractive for differentiated CLECs than for CLECs whose service offerings were homogenous, thereby explaining the market structure patterns displayed in Tables 3 and 4 . Thus, we conclude that the diffusion of CLECs into local markets throughout the United States operated through economic forces beyond scale-exceeding efficiencies. We reject the hypothesis that CLECs entry behavior is consistent with the presence of homogenous product competition. Instead, we find strong evidence consistent with the presence of customertargeted differentiation. Also, the size of the parameter estimates suggests that the motive to differentiation is economically important. Finally, different types of CLECs display distinctly different degrees of sensitivity to certain local economic conditions and to the local regulatory environment. This suggests a strong role for policy-making in shaping the development of local telecommunications markets and the services provided by the CLECs that enter. 


\section{Conclusion}

The empirical results presented here demonstrate a striking pattern of product differentiation among CLEC entrants through the late 1990s. Whether measured on the basis of target customers or geographic scope, CLECs followed entry strategies that resulted in markets that are almost evenly split between product types, as opposed to dominated by firms of one type of another. This pattern suggests that CLECs were concerned about competing with each other and that tailoring products to meet the needs of consumers was an important business strategy for them. As such, we reject the hypothesis that deregulation was driven exclusively by the logic of scale-exceeding efficiencies. Instead, we argue that differentiated behavior shaped firm entry behavior as much as differences in local economic and regulatory conditions.

We conclude that the literature on competitive local telephony should begin to investigate the many issues raised by this shift of views. The results demonstrate the role that product differentiation can play in expanding competition in previously regulated industries. For a variety of reasons, it may be difficult for a monopolist to effectively serve all types of heterogeneous customers equally well. By opening such markets up to competition, firms targeting underserved customers may enter - as they did for CLECs.

Policy makers should account for consumer welfare gains that result from better product targeting as well as from lower prices. While all pro-competitive policies for local telephony support putting entrants through a market test, our results identify what ignoring differentiation can miss. Policy making should not presume it knows the formula for commercial success solely on the basis of observing ILECs and counting the number of incumbent CLECs. Instead policy should identify CLEC strategies that differ from those of the ILEC and other CLECs, with the intent of encouraging firms that let consumers choose among an expanded array of options. 


\section{References}

Abel, Jaison. 2002. "Entry into Regulated Monopoly Markets: The Development of a Competitive Fringe in the Local Telephone Industry." Journal of Law and Economics 45, 289-316.

and Matthew Clements. 2001. "Entry Under Asymmetric Regulation.” Review of Industrial Organization 19 (2), 227-42.

Athey, Susan and Scott Stern. 1998. "An Empirical Framework for Testing Theories About Complementarities in Organizational Design," mimeo.

Berry, Steven T. 1992. "Estimation of a Model of Entry in the Airline Industry." Econometrica 60 (4), 889-917.

Bresnahan, Timothy F. and Peter C. Reiss. 1991. "Entry and Competition in Concentrated Markets." Journal of Political Economy 99 (5) 977-1009.

Crandall, Roberts W. 2001. "An Assessment of the Competitive Local Exchange Carriers five Years After the Passage of the Telecommunications Act" A Report for the USTA by Criterion Economics, LLC.

-and J. Gregory Sidek (2002), "Is Structural Separation of Incumbent Local Exchange Carriers Necessary for Competition?" Yale Journal on Regulation, 19(2), 1-75.

Federal Communication Commission. 2000. "Local Telephone Competition: Status as of June 30, 2000," Industry Analysis Division, Common Carrier Bureau, available at http://www.fcc.gov/Bureaus/Common_Carrier/Reports/FCC-State_Link/recent.html

_. 2001. "Local Telephone Competition: Status as of June 30, 2001," Industry Analysis Division, Common Carrier Bureau, available at http://www.fcc.gov/Bureaus/Common_Carrier/Reports/FCC-State_Link/recent.html

Gregg, Billy Jack. 2002. "A Survey of Unbundled Network Element Prices in the United States," Public Service Commission of West Virginia, mimeo.

Mazzeo, Michael J. 2002. "Product Choice and Oligopoly Market Structure." Rand Journal of Economics 33 (2), 221-42.

Mini, Federico. 2001. "The Role of Incentives for Opening Monopoly Markets: Comparing GTE and BOC Cooperation with Local Entrants." Journal of Industrial Economics 49 (3), 379-413.

New Resources Paradigm Group (2000), 1999 CLEC Report, $11^{\text {th }}$ edition.

Rosston, Greg, and Brad Wimmer. 2001. 'From C to Shining C' Competition and Cross-Subsidy in Communications. In Communications Policy in Transition: The Internet and Beyond, edited by Benjamin Compaine and Shane Greenstein. Cambridge, MA:MIT Press. 
Shiman, Daniel, and Jessica Rosenworcel (2002), "Assessing the Effectiveness of Section 271 Five Years After the Telecommunications Act of 1996." In Communications Policy and Information Technology: Promises, Problems and Prospects, edited by Benjamin Compaine and Shane Greenstein. Cambridge, MA: MIT Press.

Woroch, Glenn. 2001. Local Network Competition In Handbook of Telecommunications Economics, edited by Martin Cave, Sumit Majumdar, and Ingo Vogelsang. Elsevier Publishing.

Zolnierek, James, James Eisner and Eller Burton. 2001. "An Empirical Examination of Entry Patterns in Local Telephone Markets." Journal of Regulatory Economics 19 (2), 143-59. 